\title{
Some mirror partners with complex multiplication
}

\author{
Jan Christian Rohde
}

\begin{abstract}
In this note we give examples of families of Calabi-Yau 3-manifolds over Shimura varieties, whose mirror families contain subfamilies over Shimura varieties. In the case of these families we obtain dense sets of complex multiplication fibers by using the fact that the base space is a Shimura variety. In view of the work of Gukov and Vafa [7] this is of special interest in theoretical physics.
\end{abstract}

\section{Introduction}

In theoretical physics rational conformal field theories are considered as particularly interesting class of conformal field theories. Let $(\mathscr{X}, \mathscr{Y})$ be a pair of families of Calabi-Yau 3-manifolds, which are mirror partners, $X$ be a fiber of $\mathscr{X}$ and $Y$ be a fiber of $\mathscr{Y}$. In [7] Gukov and Vafa explain that $X$ and $Y$ yield a rational conformal field theory, if and only if both fibers have complex multiplication (CM). A family of Calabi-Yau manifolds over a Shimura variety has a dense set of CM fibers, if the variation of Hodge structures (VHS) is related to the Shimura datum of the base space in a natural way as in [10]. At present several of such families of Calabi-Yau 3 -manifolds over Shimura varieties are known [2, 6, 10,11,12]. In general, one does not know a Shimura subvariety of the base space on the mirror side. ${ }^{1}$ Here we give new examples of pairs of families of Calabi-Yau 3-manifolds over Shimura varieties, which are subfamilies of mirror partners.

We start with a family $\mathscr{C}_{3}$ of degree 3 covers of $\mathbb{P}^{1}$ with six different ramification points over an open Shimura subvariety $\mathscr{M}_{3} \subset\left(\mathbb{P}^{1}\right)^{3}$. By using the Fermat curve of degree 3 and $\mathscr{C}_{3}$, one can construct a family of $K 3$ surfaces with a non-symplectic involution over $\mathscr{M}_{3}$ as described in [10, Section 8]. The Borcea-Voisin construction yields a family $\mathscr{W}$ of Calabi-Yau 3-manifolds, which has a dense set of CM fibers. Garbagnati and van Geemen [5] have given a more general method to construct $K 3$ surfaces, which yields the same $K 3$ surfaces for the fibers of $\mathscr{C}_{3}$. The latter method allows one to

\footnotetext{
${ }^{1}$ Moreover, for some of these examples $[6,11]$ the existence of a mirror is not clear.
} 
construct $K 3$ surfaces with non-symplectic involutions over the boundary of $\mathscr{M}_{3} \subset\left(\mathbb{P}^{1}\right)^{3}$, where branch points of the fibers of $\mathscr{C}_{3}$ collide. Here we show that the Borcea-Voisin construction yields a family of Calabi-Yau 3-manifolds over a Shimura subvariety contained in the boundary of the base space of $\mathscr{W}$, whose fibers are its own Borcea-Voisin mirrors. Moreover, here we find a Shimura surface on the boundary of the base space of $\mathscr{W}$ such that the fibers of a family of Calabi-Yau 3-manifolds over this surface are Borcea-Voisin mirrors of the fibers of $\mathscr{W}$. We will also see that these families contain dense sets of CM fibers.

\section{Construction of $K 3$ surfaces by automorphisms}

In this section we recall the construction of $K 3$ surfaces by the methods in [5]. For this construction we use the following families of curves:

1) The family $\mathscr{C}_{1}$ is the family of genus 2 curves given by

$$
V\left(y^{3}-x_{1}\left(x_{1}-x_{0}\right)^{2}\left(x_{1}-\lambda x_{0}\right)^{2} x_{0}\right) \rightarrow \lambda \in \mathscr{M}_{1}:=\mathbb{P}^{1} \backslash\{0,1, \infty\} .
$$

2) The family $\mathscr{C}_{2}$ is the family of genus 3 curves given by

$$
V\left(y^{3}-x_{1}\left(x_{1}-x_{0}\right)\left(x_{1}-\alpha x_{0}\right)\left(x_{1}-\beta x_{0}\right) x_{0}^{2}\right) \rightarrow(\alpha, \beta) \in \mathscr{M}_{2},
$$

where

$$
\mathscr{M}_{2}:=\left(\mathbb{P}^{1} \backslash\{0,1, \infty\}\right)^{2} \backslash\{\alpha=\beta\}
$$

3) The family $\mathscr{C}_{3}$ is the family of genus 4 curves given by

$$
V\left(y^{3}-x_{1}\left(x_{1}-x_{0}\right)\left(x_{1}-\alpha x_{0}\right)\left(x_{1}-\beta x_{0}\right)\left(x_{1}-\gamma x_{0}\right) x_{0}\right) \rightarrow(\alpha, \beta, \gamma) \in \mathscr{M}_{3},
$$

where

$$
\mathscr{M}_{3}:=\left(\mathbb{P}^{1} \backslash\{0,1, \infty\}\right)^{3} \backslash(\{\alpha=\beta\} \cup\{\alpha=\gamma\} \cup\{\beta=\gamma\}) .
$$

Remark 1.1. The families $\mathscr{C}_{1}$ and $\mathscr{C}_{2}$ can be obtained by collision of the branch points of the fibers of $\mathscr{C}_{3}$ over the boundary divisor of $\mathscr{M}_{3} \subset\left(\mathbb{P}^{1}\right)^{3}$. Let $\Gamma$ denote the monodromy group of the VHS of $\mathscr{C}_{3}$. Note that for $j=$ $1,2,3$ one can apply the Deligne-Mostow theory [4] to the VHS of $\mathscr{C}_{j}$. For an overview of this topic see also [8]. Due to the Deligne-Mostow theory, the period domain of the family $\mathscr{C}_{j}$ is the complex ball $\mathbb{B}_{j}$ and $\mathscr{M}_{j}$ is a dense open subset of $\Gamma \backslash \mathbb{B}_{j}$. In this sense the base spaces $\mathscr{M}_{j}$ are modular. Moreover 
$\mathscr{M}_{2}$ and $\mathscr{M}_{1}$ are contained in the complement of $\mathscr{M}_{3}$ in $\Gamma \backslash \mathbb{B}_{3}$ (follows from [8, Theorem 3.1] and the description of the period map in [8, Section 4]).

One can also see that $\mathscr{M}_{j}$ is an open dense subset of a Shimura variety, which is a ball quotient. This can be concluded from the type of VHS of the given families (compare [10, Subsection 6.3]) and the description of such a VHS in the proof of [10, Theorem 4.4.4] in combination with the description of the period map above.

For $j=1,2,3$ and $p \in \mathscr{M}_{j}$ let $f_{j}(t) \in \mathbb{C}[t]$ be a degree 6 polynomial such that $\left(\mathscr{C}_{j}\right)_{p}$ is given by the equation $v^{3}-f_{j}(t)=0$. Moreover let $\xi=e^{2 \pi i \frac{1}{3}}$. It is clear that $\mathscr{C}_{j}$ has the $\mathscr{M}_{j}$-automorphism fiberwise given by

$$
\beta_{j}:(v, t) \rightarrow(\xi v, t)
$$

Let

$$
\mathbb{F}_{3}=V\left(y^{2} z-x^{3}-z^{3}\right) \subset \mathbb{P}^{2}
$$

be a genus 1 curve isomorphic to the Fermat curve of degree 3 and

$$
\alpha_{\mathbb{F}_{3}}: \mathbb{F}^{3} \rightarrow \mathbb{F}^{3} \text { be given by }(x: y: z) \rightarrow(\xi x: y: z) \text {. }
$$

We have chosen this explicit formula due to technical reasons. Moreover, let $S_{f_{j}}$ be a minimal model of a surface given by the Weierstrass equation

$$
Y^{2}=X^{3}+f_{j}^{2}(t)
$$

For the following lemma we will use methods, which occur already in the proof of [5, Proposition 2.2].

Lemma 1.1. The surface $S_{f_{j}}$ is a $K 3$ surface birationally equivalent to the quotient $\mathbb{F}_{3} \times\left(\mathscr{C}_{j}\right)_{p} /\left(\alpha_{\mathbb{F}_{3}}, \beta_{j}\right)$.

Proof. The rational map

$$
m_{j}: \mathbb{F}_{3} \times\left(\mathscr{C}_{j}\right)_{p} \rightarrow S_{f_{j}}
$$

is given by

$$
((t, v),(x, y)) \rightarrow\left(v^{2} x, v^{3} y, t\right)
$$


The reader checks easily that $m_{j}$ is $\left(\alpha_{\mathbb{F}_{3}}, \beta_{j}\right)$-invariant and of degree 3 . Moreover, one computes

$$
Y^{2}=\left(v^{3} y\right)^{2}=v^{6} y^{2}=v^{6}\left(x^{3}+1\right)=\left(v^{2} x\right)^{3}+f_{j}^{2}(t)=X^{3}+f_{j}^{2}(t) .
$$

From [5] we know that the minimal model $S_{f_{j}}$ is a $K 3$ surface.

\section{Some automorphisms of our $K 3$ surfaces}

2.1. The surface $S_{f_{j}}$ has an elliptic fibration given by

$$
S_{f_{j}} \rightarrow \mathbb{P}^{1} \text { via }(X, Y, t) \rightarrow t
$$

in the following way (see also [5]):

If $f_{j}\left(t_{0}\right) \neq 0$, the fiber of $t_{0}$ is given by the elliptic curve

$$
V\left(Y^{2} Z=X^{3}+u Z^{3}\right) \subset \mathbb{P}^{2}, \quad \text { where } u=f_{j}^{2}\left(t_{0}\right) .
$$

Now let $t_{0} \in \mathbb{P}^{1}$ be a zero of $f_{j}(t)$. By using the Tate algorithm, one can compute the singular fibers. If $f_{j}(t)$ has a simple zero in $t_{0}$, the singular fiber $\left(S_{f_{j}}\right)_{t_{0}}$ is of type $\mathbf{I V}$. Thus it consists of three rational curves intersecting transversally in one point.

Now assume that $f_{j}(t)$ has a double zero in $t_{0}$. Then the fiber $\left(S_{f_{j}}\right)_{t_{0}}$ is of type $\mathbf{I V}^{*}$. Thus it is given by seven rational curves with the following intersection graph of type $\tilde{E}_{6}$ :

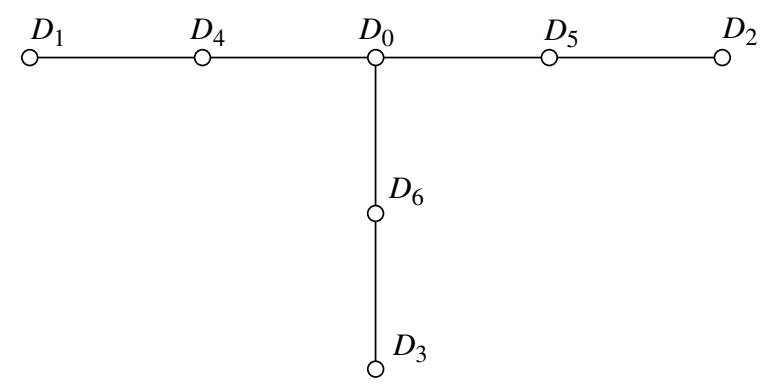

Let $\iota_{f_{j}}: S_{f_{j}} \rightarrow S_{f_{j}}$ denote the involution given by

$$
(X, Y, t) \rightarrow(X,-Y, t)
$$

and $\alpha_{f_{j}}: S_{f_{j}} \rightarrow S_{f_{j}}$ denote the automorphism of degree 3 given by

$$
(X, Y, t) \rightarrow(\xi X, Y, t) .
$$


2.2. The fixed locus of $\iota_{f}$ contains clearly the section $s_{\infty}$ of the elliptic fibration fiberwise given by $(0: 1: 0) \in \mathbb{P}^{2}$ for a general $t \in \mathbb{P}^{1}$ and the curve $C_{j, p}$ given by

$$
X^{3}+f_{j}^{2}(t)=0
$$

which is isomorphic to $\left(\mathscr{C}_{j}\right)_{p}$ (see $[10$, Remark 2.1.8]). One can also verify by explicit computation that one obtains a fiber $F$ of type IV over a simple zero of $f_{j}(t)$ by blowing up once. This computation shows that $\iota_{f_{j}}$ interchanges two irreducible components of $F$ and $C_{j, p}$ intersects $F$ in the intersection point of its irreducible components. The involution $\iota_{f_{j}}$ acts non-trivially on the third irreducible component of $F$ with isolated fixed point given by the intersection point with $s_{\infty}$.

Moreover, the fixed locus of the automorphism $\alpha_{f_{j}}$ contains the sections

$$
s_{ \pm}(t)=\left(0: \pm f_{j}(t): 1\right) \in \mathbb{P}^{2}
$$

One can assume that $s_{\infty}$ is the zero-section and under this assumption one can easily check that $s_{ \pm}$are sections of 3 -torsion points by considering the generic fiber. The algebraic group on the special fiber $F^{*}$ of type $\mathbf{I V}^{*}$ is given by

$$
\left(D_{1} \backslash D_{4}\right) \cup\left(D_{2} \backslash D_{5}\right) \cup\left(D_{3} \backslash D_{6}\right) \cong \mathbb{G}_{a, \mathbb{C}} \times \mathbb{Z}_{3}
$$

Thus one has three different 3-torsion points such that each connected component contains precisely one 3 -torsion point. Due to the Neron property, the sections $s_{ \pm}$and $s_{\infty}$ intersect $F^{*}$ in the 3 -torsion points. Thus we can assume without loss of generality that $s_{\infty}$ intersects $D_{3} \backslash D_{6}$, the section $s_{+}$ intersects $D_{1} \backslash D_{4}$ and $s_{-}$intersects $D_{2} \backslash D_{5}$.

Since the fixed locus of $\iota_{f_{j}}$ contains curves, $\iota_{f_{j}}$ is non-symplectic. Recall that a non-symplectic involution of a $K 3$ surface has a fixed locus, which is either empty or consists of smooth disjoint curves (see [3]). For a general description of automorphisms of K3 surfaces see [1]. Thus from the graph of a fiber of type $\mathbf{I V}^{*}$ one concludes:

Proposition 2.1. The curve $D_{6}$ is contained in the fixed locus with respect to $\iota_{f_{j}}$ and $\iota_{f_{j}}$ interchanges the handle consisting of $D_{1}$ and $D_{4}$ with the handle consisting of $D_{2}$ and $D_{5}$. Moreover, the intersection point $F^{*} \cap C_{j, p}$ is the additional isolated fixed point of $\iota_{f_{j}} \mid D_{0}$. 


\section{Construction of mirror pairs with complex multiplication}

Recall 3.1. Let $S$ be a $K 3$ surface with non-symplectic involution $\iota_{S}$, which has a fixed locus consisting of the curves $C_{1}, \ldots, C_{N}$, and $E$ be an elliptic curve with involution $\iota_{E}$ fixing four points. Moreover, let

$$
N^{\prime}=\sum_{i=1}^{N} g\left(C_{i}\right),
$$

where $g\left(C_{i}\right)$ denotes the genus of $C_{i}$. Then the Calabi-Yau 3-manifold $X$ obtained from the Borcea-Voisin construction given by blowing up the singularities of $S \times E /\left(\iota_{S}, \iota_{E}\right)$ once has the Hodge numbers

$$
h^{1,1}(X)=11+5 N-N^{\prime} \quad \text { and } \quad h^{2,1}(X)=11+5 N^{\prime}-N
$$

(see [13]).

In many cases, the action of $\iota_{S}$ on the integral cohomology lattice $H^{2}(S, \mathbb{Z})$ of $S$ can be used to construct a second involution $\iota^{\prime}$ on the same lattice. The involution $\iota^{\prime}$ can be realized as an involution of a family of $K 3$ surfaces $\mathscr{S}^{\prime} \rightarrow \mathscr{B}$ over $\mathscr{B}$, whose restrictions to each fiber of $\mathscr{S}^{\prime}$ are non-symplectic involutions. By a relative version of the construction above for $\mathscr{S}^{\prime}$, one obtains the Borcea-Voisin mirror family of $X$ (for details see $[3,13]$ ).

Remark 3.2. By using $\mathscr{C}_{3}$, one has already constructed families of CalabiYau manifolds over Shimura varieties (see [10, Section 8 and Section 9]). For this construction in [10] one has used a family of $K 3$ surfaces obtained from a tower of cyclic coverings of weighted projective spaces $\mathbb{P}(2,2,1, \ldots, 1)$. The family of $K 3$ surfaces is precisely the family over $\mathscr{M}_{3}$, which occurs in $[5$, Remark 1.3] and also here. This follows from the fact that both constructions yield a $K 3$ surface, which is a minimal model of $\mathbb{F}_{3} \times\left(\mathscr{C}_{j}\right)_{p} /\left(\alpha_{\mathbb{F}_{3}}, \beta_{j}\right)$ (see $[10$, Section 8.2]).

In [10] the fibers of the family of $K 3$ surface come with an embedding into a desingularization of the weighted projective space $\mathbb{P}(2,2,1,1)$ given by

$$
\begin{aligned}
\tilde{\mathbb{P}}(2,2,1,1) & \ni \tilde{V}\left(y_{2}^{3}+y_{1}^{3}+x_{1}\left(x_{1}-x_{0}\right)\left(x_{1}-a x_{0}\right)\left(x_{1}-b x_{0}\right)\left(x_{1}-c x_{0}\right) x_{0}\right) \\
& \rightarrow(a, b, c) .
\end{aligned}
$$

The non-symplectic involution $\gamma$, which is used in [10] for a Borcea-Voisin construction, is obtained from the involution of $\tilde{\mathbb{P}}(2,2,1,1)$ given by $y_{2} \leftrightarrow y_{1}$. Thus one checks easily that $\gamma$ generates the Galois group of a Galois cover 
of degree 2 onto the Hirzebruch surface

$$
H_{2} \cong \tilde{V}\left(y_{2}-y_{1}\right) \subset \tilde{\mathbb{P}}(2,2,1,1)
$$

with ramification divisor isomorphic to a disjoint union of $\mathbb{P}^{1}$ and $C_{j, p}$. Thus from [9], the fact that the ramification divisor of the quotient by $\iota_{f_{3}}$ is isomorphic to the ramification divisor of the quotient by $\gamma$ tells us that both coverings are coverings onto $\mathrm{H}_{2}$. Since the branch divisors in $\mathrm{H}_{2}$ coincide up to isomorphism, $\gamma$ and $\iota_{f_{3}}$ coincide also and yield isomorphic families of Calabi-Yau 3-manifolds by the Borcea-Voisin construction.

3.3. By 2.2, the involution $\iota_{f_{j}}$ on $S_{f_{j}}$ has a fixed locus containing a rational curve $s_{\infty}$ and the curve $C_{j, p}$ of genus $j+1$. Moreover, the elliptic fibration of $S_{f_{j}}$ contains $3-j$ singular fibers of type $\mathbf{I V}^{*}$ and each of these fibers has one rational curve contained in the fixed locus of $\iota_{f_{j}}$ (see Proposition 2.1). Thus by using (1) and the family of elliptic curves

$$
\mathscr{E} \rightarrow \mathscr{M}_{1}, \quad V\left(y^{2} z-x(x-z)(x-\lambda z)\right) \rightarrow \lambda,
$$

the Borcea-Voisin construction yields families $\mathscr{X}_{j} \rightarrow \mathscr{M}_{j} \times \mathscr{M}_{1}$ of CalabiYau 3-manifolds with the following Hodge numbers:

\begin{tabular}{|c||c|c|}
\hline$j$ & $h^{1,1}$ & $h^{2,1}$ \\
\hline \hline 3 & 17 & 29 \\
\hline 2 & 23 & 23 \\
\hline 1 & 29 & 17 \\
\hline
\end{tabular}

Remark 3.4. By [3, Section 3 and 4], one can easily check that $\mathscr{X}_{2}$ is contained in a family, which is its own Borcea-Voisin mirror family. Moreover, the families $\mathscr{X}_{1}$ and $\mathscr{X}_{3}$ can be embedded in families, which are BorceaVoisin mirrors of each other.

Remark 3.5. By the construction above, the families $\mathscr{X}_{1}$ and $\mathscr{X}_{2}$ are contained in the boundary of $\mathscr{X}_{3}$. Moreover, by using Remark 1.1, one can show that the period map of $\mathscr{X}_{j}$ is a multivalued map to a dense open subset of $\mathbb{B}_{j} \times \mathbb{B}_{1}$. From these results one can conclude that the base space of $\mathscr{X}_{j}$ is an open subset of a Shimura variety with associated Hermitian symmetric domain $\mathbb{B}_{j} \times \mathbb{B}_{1}$.

By analogous arguments, one can also see that $\mathscr{X}_{1}$ is defined over the boundary of $\mathscr{X}_{2}$. 
By $[13,2.21]$, we have a precise description of the mirror map for the families $\mathscr{X}_{j}$ and $\mathscr{X}_{4-j}$. Due to [7] one can assume that each pair of complex multiplication fibers of $\mathscr{X}_{j}$ and $\mathscr{X}_{4-j}$ yields a rational conformal field theory. Now we are going to show that each $\mathscr{X}_{j}$ has a dense set of CM fibers for $j=1,2,3$. First we introduce our definition of CM:

3.6. Let $X$ be a compact Kähler manifold of complex dimension $n$ and $S^{1}$ be the $\mathbb{R}$-algebraic group

$$
S^{1}=\operatorname{Spec}\left(\mathbb{R}[x, y] / x^{2}+y^{2}-1\right),
$$

where

$$
S^{1}(\mathbb{R})=\left\{M=\left(\begin{array}{cc}
a & b \\
-b & a
\end{array}\right) \in \mathrm{SL}_{2}(\mathbb{R})\right\} \cong\{z \in \mathbb{C}:|z|=1\} .
$$

The rational Hodge structure on $H^{n}(X, \mathbb{Q})$ of weight $n$ corresponds to the representation

$$
\begin{aligned}
& h_{X}: S^{1} \rightarrow \operatorname{GL}\left(H^{n}(X, \mathbb{R})\right), \quad h_{X}(z) v=z^{p} \bar{z}^{q} v \quad\left(\forall v \in H^{p, q}(X)\right. \\
& \text { with } p+q=n) .
\end{aligned}
$$

Since $H^{p, q}(X)=\overline{H^{q, p}(X)}$, one can see that $h_{X}\left(S^{1}\right) \subset \mathrm{GL}\left(H^{n}(X, \mathbb{R})\right)$. The Hodge group $\operatorname{Hg}(X)$ is the smallest $\mathbb{Q}$-algebraic subgroup $G$ of $\operatorname{GL}\left(H^{n}(X, \mathbb{Q})\right)$ such that $h_{X}\left(S^{1}\right) \subset G_{\mathbb{R}}$. We say that $X$ has $\mathrm{CM}$, if $\mathrm{Hg}(X)$ is a torus.

For more details in the case of Calabi-Yau 3-manifolds see also [2].

Remark 3.7. Now let $X$ be a Calabi-Yau 3-manifold. In another definition, which is also used in [7], the Calabi-Yau manifold $X$ has complex multiplication, if the torus

$$
J_{G}(X)=H^{3}(X, \mathbb{Z}) \backslash H^{n}(X, \mathbb{C}) / F^{2}\left(H^{3}(X, \mathbb{C})\right)
$$

is of CM type. The Griffiths intermediate Jacobian $J_{\mathrm{G}}(X)$ is of CM type, if and only if the endomorphism algebra of $J_{\mathrm{G}}(X)$ has dimension $2\left(H^{2,1}(X)+1\right)$. By [2, Theorem 2.3], a Calabi-Yau 3-manifold with CM in the sense of 3.6, which is also the same definition of CM introduced by Borcea in [2], satisfies that $J_{\mathrm{G}}(X)$ is of CM type. ${ }^{2}$

\footnotetext{
${ }^{2}$ It is a common mistake to claim that the both definitions of complex multiplication would be equivalent. The assumption that $J_{\mathrm{G}}(X)$ is of CM type does not imply that $X$ has CM in the sense of 3.6. For a counter example see [10, Example $1.6 .9]$.
} 
Proposition 3.1. For $j=1,2,3$, the family $\mathscr{X}_{j}$ has a dense set of $C M$ fibers.

Proof. By [10, Subsection 6.3], each family $\mathscr{C}_{j}$ has a dense set of CM fibers. Note that the family of elliptic curves

$$
\mathscr{E} \rightarrow \mathscr{M}_{1}, \quad V\left(y^{2} z-x(x-z)(x-\lambda z)\right) \rightarrow \lambda
$$

has also a dense set of CM fibers. Since the ramification locus of the involution $\iota_{f_{j}}$ on $S_{f_{j}}$ consists of $C_{j, p} \cong\left(\mathscr{C}_{j}\right)_{p}$ and some rational curves, it remains to show that $S_{f_{j}}$ has CM, if $\left(\mathscr{C}_{j}\right)_{p}$ has CM. Using this result one can then conclude as in $[10$, Subsection 7.2$]$ that $\left(\mathscr{X}_{j}\right)_{(p, q)}$ has CM, if $\left(\mathscr{C}_{j}\right)_{p}$ and $\mathscr{E}_{q}$ have CM.

The singularities of the fibers of $\mathbb{F}_{3} \times \mathscr{C}_{j} /\left(\alpha_{\mathbb{F}_{3}}, \beta_{j}\right)$ are given by the singular sections. Let $m_{j}$ denote the quotient map by $\left(\alpha_{\mathbb{F}_{3}}, \beta_{j}\right)$. Near the sections of fixed points corresponding to the singular sections of $\mathbb{F}_{3} \times \mathscr{C}_{j} /\left(\alpha_{\mathbb{F}_{3}}, \beta_{j}\right)$ the action of $\left(\alpha_{\mathbb{F}_{3}}, \beta_{j}\right)$ is given by $(\xi, \xi)$ or $(\xi, \bar{\xi})$.

First consider the case $(\xi, \bar{\xi})$. In this case one blows up the corresponding sections on $\mathbb{F}_{3} \times \mathscr{C}_{j}$ with exceptional divisor $E_{1}$. The automorphism $\left(\alpha_{\mathbb{F}_{3}}, \beta_{j}\right)$ does not act trivially on $E_{1}$. Thus we blow up the two fixed sections on each connected component of $E_{1}$ with smooth exceptional divisor $E_{2}$. This divisor is contained in ramification locus of $m_{j}$. Now the quotient by $\left(\alpha_{\mathbb{F}_{3}}, \beta_{j}\right)$ is smooth in a neighborhood of $m_{j}\left(E_{1} \cup E_{2}\right)$.

In the case $(\xi, \xi)$ we blow up the section of fixed points and obtain a smooth exceptional divisor contained in the ramification locus.

Let $\widehat{\mathbb{F}_{3} \times \mathscr{C}_{j}}$ denote the manifold obtained from the previous blowing up operations on $\mathscr{C}_{j} \times \mathbb{F}_{3}$ and

$$
\mathscr{F}_{j}=\widetilde{\mathbb{F}_{3} \times \mathscr{C}_{j}} /\left(\alpha_{\mathbb{F}_{3}}, \beta_{j}\right)
$$

Thus we obtain a model $\mathscr{F}_{j}$ of the quotient $\mathbb{F}_{3} \times\left(\mathscr{C}_{j}\right)_{p} /\left(\alpha_{\mathbb{F}_{3}}, \beta_{j}\right)$ consisting of smooth fibers over $\mathscr{M}_{j}$. The surface $\mathbb{F}_{3} \times \mathscr{C}_{j}$ has $\mathrm{CM}$, if $\mathbb{F}_{3}$ and $\left(\mathscr{C}_{j}\right)_{p}$ have $\mathrm{CM}$. Note that monoidal transformations of surfaces do not have any effect to the property of CM (compare [10, Corollary 7.1.6]). Due to the fact that the Hodge structure on $H^{2}\left(\left(\mathscr{F}_{j}\right)_{p}, \mathbb{Q}\right)$ is a sub-Hodge structure of the one on $H^{2}\left(\left({\widetilde{\mathbb{F}_{3} \times \mathscr{C}_{j}}}_{p}, \mathbb{Q}\right)\right.$, one concludes that $\left(\mathscr{F}_{j}\right)_{p}$ has $\mathrm{CM}$, if $\left(\mathscr{C}_{j}\right)_{p}$ has CM. Moreover, we can use monoidal transformations to obtain $S_{f_{j}}$ from $\left(\mathscr{F}_{j}\right)_{p}$. Thus $S_{f_{j}}$ has CM, if $\left(\mathscr{F}_{j}\right)_{p}$ has CM. 


\section{Acknowledgments}

This paper was written at the Graduiertenkolleg "Analysis, Geometry and String Theory" at Leibniz Universität Hannover. I would like to thank Lars Halle for instructive discussions about Neron models and for once asking the question "What happens on the boundary of $\mathscr{W} ? "$. This question is partially answered here. I would like to thank Klaus Hulek and Matthias Schütt for their interest and their comments, which helped to improve this text. Moreover, I would like to thank Bernd Siebert, who has pointed out that it is of interest to understand the period maps of the given families, which are outlined in Remark 1.1 and Remark 3.5. I would also like to thank Noriko Yui for usefull comments. In addition, I would like to thank one of the referees of this paper whose comments helped to simplyfy the computation of the fixed loci of the non-symplectic involutions on the $K 3$ surface here.

\section{References}

[1] M. Artebani, A. Sarti and S. Taki, K3 surfaces with non-symlectic automorphisms of prime order, DOI 10.1007/s00209-010-0681-x, to appear in Math. Z.

[2] C. Borcea, Calabi-Yau threefolds and complex multiplication. Essays on mirror manifolds, Internat. Press, Hong Kong, 1992, 489-502.

[3] C. Borcea, K3 Surfaces with involution and mirror pairs of Calabi-Yau manifolds, in 'Mirror symmetry II', AMS/IP, Providence, RI, 1997, 717-743.

[4] P. Deligne and G. Mostow, Monodromy of hypergeometric functions and non-lattice integral monodromy, IHES 63 (1986) 5-89.

[5] A. Garbagnati and B. van Geemen, The Picard-Fuchs equation of a family of Calabi-Yau threefolds without maximal unipotent monodromy, Int. Math. Res. Not. 16 (2010), 3134-3143.

[6] A. Garbagnati, New families of Calabi-Yau 3-folds without maximal unipotent monodromy, 2010, arXiv:1005.0094.

[7] S. Gukov and C. Vafa, Rational conformal field theories and complex multiplication, Comm. Math. Phys. 246 (2004), 181-210.

[8] E. Looijenga, Uniformization by Lauricella functions: an overview of the theory of Deligne Mostow, in 'Arithmetic and Geometry Around Hypergeometric Functions', eds. Rolf-Peter Holzapfel, A. Muhammed Uludag, 
and Masaaki Yoshiba, Progress in Mathematics, 260, Birkhäuser, Basel, 2007, 207-244.

[9] V. V. Nikulin, Discrete reflection groups in Lobachevsky spaces and algebraic surfaces, in 'Proc. ICM', Berkeley, California, 1986, 654-671.

[10] J. C. Rohde, Cyclic coverings, Calabi-Yau manifolds and complex multiplication, Lecture Notes in Mathematics, 1975, Springer-Verlag, Berlin, Heidelberg, 2009.

[11] J. C. Rohde, Maximal automorphisms of Calabi-Yau manifolds versus maximally unipotent monodromy, Man. Math. 131 (2010), 459-474.

[12] E. Viehweg and K. Zuo, Complex multiplication, Griffiths-Yukawa couplings, and rigidity for families of hypersurfaces, J. Alg. Geom. 14 (2005), 481-528.

[13] C. Voisin, Miroirs et involutions sur les surfaces K3, J. Géom. Algébrique d'Orsay, Asterisque 218 (1993), 273-323.

UNIVERSITÄT HAMBURG

BUNDESSTR. 55

20146 HAMBURG

GERMAny

E-mail address: jan.christian.rohde@math.uni-hamburg.de

Received June 25, 2010

The paper is dedicated to Eckart Viehweg. 
